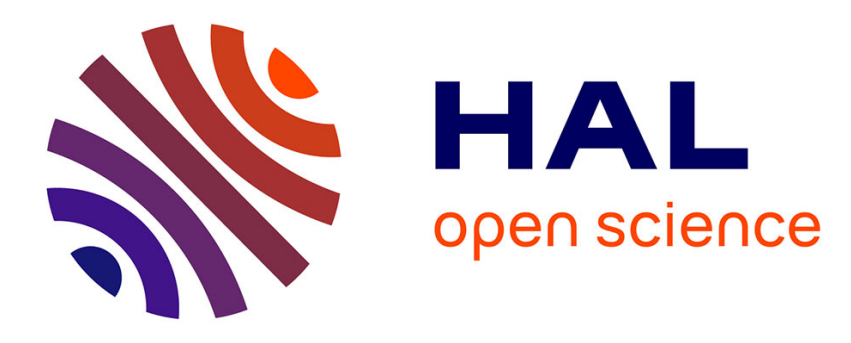

\title{
Modeling pavlovian conditioning with multiple neuronal populations
}

Maxime Carrere, Frédéric Alexandre

\section{To cite this version:}

Maxime Carrere, Frédéric Alexandre. Modeling pavlovian conditioning with multiple neuronal populations. IEEE International Joint Conference on Neural Networks, Jul 2015, Killarney, Ireland. 10.1109/IJCNN.2015.7280716 . hal-01237877

\section{HAL Id: hal-01237877 \\ https://hal.inria.fr/hal-01237877}

Submitted on 3 Dec 2015

HAL is a multi-disciplinary open access archive for the deposit and dissemination of scientific research documents, whether they are published or not. The documents may come from teaching and research institutions in France or abroad, or from public or private research centers.
L'archive ouverte pluridisciplinaire HAL, est destinée au dépôt et à la diffusion de documents scientifiques de niveau recherche, publiés ou non, émanant des établissements d'enseignement et de recherche français ou étrangers, des laboratoires publics ou privés. 


\title{
Modeling pavlovian conditioning with multiple neuronal populations
}

\author{
Maxime Carrere and Frédéric Alexandre \\ LaBRI, Université de Bordeaux, Institut Polytechnique de Bordeaux, \\ CNRS, UMR 5800, Talence, France \\ Inria Bordeaux Sud-Ouest, Talence, France \\ Institut des Maladies Neurodégénératives, Université de Bordeaux, \\ CNRS, UMR 5293, Bordeaux, France
}

\begin{abstract}
Artificial Neural Networks are often used as black boxes to implement behavioral functions, developed by trials and errors, fed with sensory inputs and controlled by some criteria of performance. This is the case for pavlovian conditioning where important sensory information is non ambiguous and where the error of prediction is to be minimized. These past years, taking into account critical conditioning behaviors entailed complexifying the neuronal functioning and learning rules. This resulted in networks still simple at the architectural level but with a dynamics difficult to master. Instead, we propose a new neuronal model using uniform and classical neuronal dynamics, with a more complex architecture based on recent findings in neuroscience. Results reported in this paper confirm the good behavior of the model and justify the complex architecture by the greater robustness and flexibility of the model.
\end{abstract}

\section{INTRODUCTION TO PAVLOVIAN CONDITIONING}

Many models of Artificial Neural Networks have been proposed to implement pavlovian conditioning. One reason is that this topic is attractive because pavlovian conditioning is a learning process easily observable at the behavioral level in most species, from the simplest to the most complex, and that it involves stimuli easy to observe and to control in the environment. Another reason is that pavlovian conditioning is a gateway to fundamental concepts like prediction error, attention and information representation, as it has been shown by the variety of topics addressed by these models. Consequently most of the models that have been presented to date have the double characteristic of firstly proposing a simple implementation to capture the essential features of pavlovian conditioning and secondly complexifying the model to stick to a more realistic view of this process [1], [2], [3], [4], [5], [6], [7], [8].

Pavlovian conditioning is the adaptive process by which a biologically significant stimulus, also known as unconditional stimulus (US), can be anticipated by learning by a neutral stimulus, also known as conditioned stimulus (CS). US can correspond to a reward (some food) or to a punishment (an electric shock) and automatically triggers a response, not only at the motor level (e.g. orientation, avoidance) but also at more internal hormonal and autonomic levels to prepare the body (e.g. release of stress hormones, acceleration of heart rate). After some pairings between CS and US, the association CSUS is learnt and the CS presented alone will trigger a pavlovian response.

From a modeling point of view, this simple description might evoke a simple associative rule between CS and US, except if other phenomena like blocking are considered. In the blocking protocol, also described as a principle of parsimony, a stimulus CS1 is paired with a US until acquisition of the association. Then, the conjunction of two stimuli CS1 and CS2 is paired with the same US. After this process, it appears that CS1 but not CS2 predicts the US. This can be interpreted as a parsimonious learning, since in the learnt cases, CS1 is sufficient to predict US and there is no need to learn on CS2. In the early 70's, Rescorla and Wagner [9] brought a decise answer to this problem, proposing that the associative strength between CS and US was in fact proportional to the degree of surprise or of impredictibility of what happens. [10] proposed a simple implementation of their rule in a perceptron-like neural network. In this network, each possible $C S_{j}$ activates a neuron in an input layer, which is directly connected through a weighted link $W_{i j}$ to an output unit predicting $U S_{i}$. The Rescorla-Wagner learning rule is implemented as:

$$
\Delta W_{i j}=\alpha * \beta *\left(U S_{i}-\Sigma_{j} W_{i j} * C S_{j}\right) * C S_{i j}
$$

where $\alpha$ is a parameter standing for the associability (saliency) of the CS, $\beta$ for the effectiveness (behavioral importance) of the US and $\left.\Sigma W_{i j} * C S_{j}\right)$ is the prediction of $U S_{i}$ by the network. Consequently, the term $\left(U S_{i}-\Sigma W_{i j} * C S_{j}\right)$ can be seen as the error of prediction of the network, which is a major ingredient in most approaches of reinforcement learning. Sutton and Barto [6] have introduced a temporal dimension to this rule (the CS must begin before the US), using temporal traces yielding residual neuronal activities when the stimulus has disappeared.

Even if the Rescorla-Wagner (RW) rule remains of central interest for modeling pavlovian conditioning, most models that were published subsequently consisted in considering some protocols where that rule cannot explain all the observed phenomena and in proposing a modification of the rule accordingly. This has been the case for several kinds of problems. The first kind of problem is about the way stimuli are represented. Learning can become complex when a configuration of stimuli requires a specific answer by the network. This is the case for example for compound stimuli (each of CS1 and CS2 in isolation predicts the US but not CS1\&CS2 together: this is the classical problem of the XOR). This is also the case when all the surrounding stimuli must be aggregated in a global 
pattern called context and when the response of the network is context dependent (it has for example been observed that the spatial context of conditioning, the box in which the rat receives electric shocks, can become a strong predictor of the US [11]). In all these cases, where the RW rule cannot learn to predict the US from an input layer made of elemental CSs, several extensions have been proposed [8], adding new units in the input layer, corresponding to configural stimuli or to the context.

Other kinds of problems are related to the mechanics of the learning process. The RW rule states that the modification of the weights depends only on the presence of CS and US. This might be modulated by parameters $\alpha$ and $\beta$, but they are supposed to be constant in the rule. In other words, according to the RW rule, learning to predict the US does not depend on the history of conditioning, which is not what is observed experimentally. Two modeling paths have been developed to take this problem into account. The Pearce-Hall model [7] measures the rate with which each stimulus is learned. This is called salience associability and corresponds to rendering parameter $\alpha$ variable and dependent on the history of each CS. A simple implementation is to multiply the RW rule by $a b s\left(U S_{i}-\Sigma W_{i j}\right)$, where abs is the absolute value function. In this case, and as it is observed experimentally, weights from CSs that already predict US a lot are modified more slowly. The Mackintosh model [4] gives a report on attentional associability which explains which stimuli have access to the learning process. In this model, good predictors of US maintain a high associability, to explain for example the fact that assigning a CS to a new US is quicker after an overtaining of the CS with previous US.

Both models underline the need to take into account the recent history of conditioning (it can be mentioned that configuration of stimuli can develop their own associability [12]) but they also result in a complexification of the learning rules. The best illustration is probably with so-called hybrid models [13] which propose to incorporate in one rule all the elements mentioned above.

Another important problem with the RW rule is the fact that it corresponds to acquiring a static and deterministic view of the world, whereas it can be stochastic (a CS can predict a US with a certain probability) and changing (a CSUS rule can be valid one day and disappear the day after), both concepts referred to, respectively, as known and unknown uncertainty [14]. The RW rule or the Pearce-Hall model can compute the probability of known uncertainty as the value of convergence of the weight, but the unknown uncertainty can only correspond to an estimation subject to changes at any moment. Consequently, a special care must be taken for unknown uncertainty, which is well represented in the case of extinction. In this protocol [15], a CS-US association is learned until the CS faithfully predicts the US; then the US in no longer given (extinction of the association). It is then observed that the prediction will disappear but this takes a rather long time (for example in fear conditioning, a CS-US association can be learned after three pairing but more than ten are necessary for extinction). More important is the related phenomenon of renewal: if after the extinction, the US is given again, the CS-US association is re-established immediately. This experiment is a strong argument against models based on the RW rule where the level of CS-US association is fluctuating with the level of association and is ultimately removed after extinction. Instead, it proposes that the CS-US association is not forgotten but only inhibited during extinction and that renewal consists in releasing that inhibition [16]. Consequently, whereas some fluctuation, as proposed in the RW rule, can be used to precisely define the level (the probability) of CS-US association, another mechanism must be defined in the case of unknown uncertainty, when the rule changes.

In summary, this overview underlines the two main characteristics of modeling pavlovian conditioning with ANNs. On the one hand, the RW rule has had a deep impact on the models and confirms the preeminence of the error of prediction of the US in pavlovian conditioning. On the other hand, adapting the RW rule to a more precise modeling of the behavioral characteristics reveals difficult in the too simple framework of a two-layered network, directly predicting the US from the CS. A key issue in this regard is to take a more insightful view of the biological substrate of this learning process, which is not limited to a two layered structure, and to develop more biologically inspired neuronal networks.

\section{CEREBRAL IMPLEMENTATION OF PAVLOVIAN CONDITIONING}

Except for the case of simple somatomotor associations where the cerebellum is mainly involved [17], another cerebral structure, the amygdala, is reported to be the key region where pavlovian conditioning takes place [18]. In this regard, two main regions have been traditionally described: the basolateral nuclei (BLA) receiving the sensory input and the central nuclei $\mathrm{CeA}$ for the motor output. More precisely, it has been shown that the CS and the US activates neurons in BLA and that the CS-US association is learned herein [19]. Similarly, strong experimental evidences have shown that CeA receives information from BLA and projects to structures responsible for the motor, hormonal and autonomic expression of pavlovian responses [20].

Concerning phenomena evoked above and indicating that pavlovian conditioning is more complex than a simple associative rule between elemental CS and US, effects related to configural or contextual representations are generally attributed to the hippocampus [21], known to build arbitrary relations among separate features of a behavioral episode and projecting to BLA. On this basis, bio-inspired models of pavlovian conditioning able to process configural and contextual stimuli have been proposed, including a model of the hippocampus dedicated to the formation of complex patterns acting as CSs [1], [2], [22], [23].

The prefrontal cortex is undoubtly considered as a major cerebral structure involved in temporal representations, including storage of past activities in working memory and behavioral sequence representation [24]. Concerning temporal aspects evoked above for pavlovian conditioning, the infralimbic cortex or the homologous structure in primates, the orbitofrontal cortex, ventral parts of the medial prefrontal cortex, have been reported to act as a working memory, keeping track of the recent reward history [25]. It has been specifically reported that projections from this structure toward BLA are necessary for the acquisition of extinction and for extinction memory [26]. Accordingly, bio-inspired neural models 
of extinction have been proposed [27], [28], implementing an inhibitory role of the medial prefrontal cortex toward CS-US associations in the amygdala in case of extinction, measured as a frequency of prediction of the US not followed by its occurrence.

In their paper describing the role of uncertainty in decision making [14], Yu and Dayan underline the prominent role of neuromodulation, specifically proposing the influence of acethylcholine and norepinephrine on the neural substrate to modulate its activity, respectively as a function of known and unknown uncertainty. As far as pavlovian conditioning is concerned, mainly the effect of acetylcholine has been considered in bio-inspired models [29], underlying that its release from the basal forebrain is partly due to hormonal responses emitted from $\mathrm{CeA}$ in case of errors of prediction and modeling its role in changing attentional and learning effects in the cortex and the hippocampus.

Whereas these bio-inspired neuronal models bring valuable insights about mechanisms underlying some complex aspects of pavlovian conditioning, they say few about how all these mechanisms coexist and interact in amygdalar neural populations. Particularly, contradictory information might be sent to BLA from its afferent structures and it might be questioned how they are incorporated at the neuronal level to elaborate the more adapted response. Solutions like complex rules proposed by hybrid models [13] do not seem transposable here for at least two reasons. They are too complex to be implemented as a neuronal activation or learning rule and they are not flexible enough to change autonomously, depending on the changes in the statistics of external events.

The model that we present in this paper addresses this problem by incorporating recent findings from neuroscience in a novel bio-inspired model of the amygdala. In short, the main characteristics that we extract from these recent findings is that distinct populations of amygdalar neurons receive distinct afferences from the neuronal structures providing key information and underlie distinct evaluations, which are combined and confronted internally in the amygdala to prepare the pavlovian response.

More precisely, the distributed neuronal implementation that we propose in our model relies on the following biological hints. The basolateral nuclei of the amygdala can in fact be differenciated as a lateral nucleus, receiving elemental sensory information from the thalamus and the cortex and a basal nucleus, receiving more elaborated information from the hippocampus and the prefrontal cortex [30]. Besides, within the basal nucleus, it has been shown recently [15] that two distinct populations of neurons, called high-fear and low-fear neurons are respectively connected with the hippocampus and the infralimbic cortex and are respectively engaged in contextdependent acquisition and renewal (high-fear neurons) and in extinction (low-fear neurons), whereas fear neurons in the lateral nucleus can detect simple CS from elemental sensory information from the thalamus and the cortex. In addition, inhibitory connections are also reported between these populations [31]. Another key difference between the basal and lateral nucleus exploited by our model is that the activity of the basal nucleus is particularly modulated by acetylcholine. For example, in [32] a higher activation in the basal nucleus is associated to a high level of acetylcholine.
A functional differentiation has also been recently reported in the lateral part of the central nucleus, concerning the preparation of the pavlovian response [33]. It is reported in this paper that in this region in mice, $30 \%$ of the neurons (called CeLon) acquire an excitatory response and $25 \%$ a strong inhibitory response and are together in mutual inhibition. It can be consequently hypothetized that fear and low-fear neurons from the lateral and basal nuclei respectively project on these populations, whose interaction results in the level of pavlovian response emitted from the central nucleus.

In summary, whereas most models of the amygdala postulate a simple network, directly connecting a sensory layer to a motor layer and managing the complex features of pavlovian conditioning by the design of a complex learning rules, biological evidences rather propose to consider that several populations coexist in the amygdala to process different classes of sensory and motor patterns, in close relation to the different classes of afferences received by the amygdala. Several consequences can be drawn from this global picture. Within such a distributed network, local learning and functioning rules can remain simple. The global behavior of the network can be more flexible, since several scenarios can be evaluated in parallel, before the decision emerges from their competition and coordination. This is more precisely described in the model that we present now.

\section{MODEL DESCRIPTION}

Our model aims at illustrating how connections in a neural network can explain and reproduce behavioral results observed in pavlovian conditioning. Thus, it describes the amygdala by modeling its differents parts using different neural populations. Each population has mainly the same neuronal equations and parameters, but differs one from the other by its connectivity, ie. differences in inputs from other populations or from neuromodulation, and differences in output projections toward other populations.

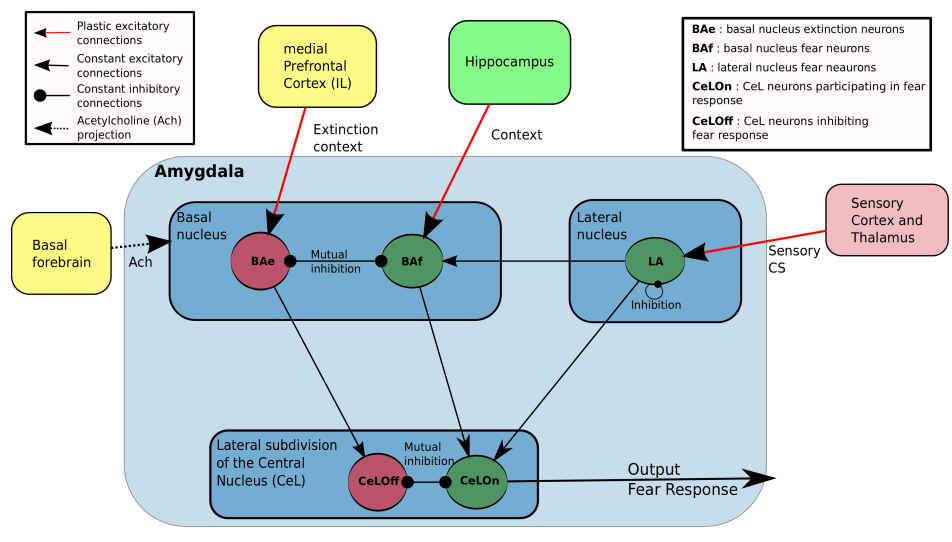

Fig. 1. Main features of our amygdalar network. LA and BAf are two fear neurons populations, which differ by their afferent connections. LA learns to predict fear, based on sensory input from cortex and thalamus and projects to BAf and CeLOn. BAf receives contextual inputs from Hippocampus and sensory-based prediction from LA, resulting in a prediction based on both sensory or contextual information. BAe is a population of extinction neurons receiving contextual inputs from medial Prefrontal Cortex (Infralimbic cortex, IL) during extinction. BAe and BAf are in mutual inhibition and respectively project to CeLOn and CeLOff populations in CeA. CeLOn and CeLOff are also in mutual inhibition. CeLOn activity is considered as the level of US prediction of the model. 
As described in figure 1, the amygdala is represented by five different populations, and encompasses three main information flows. First, a population of fear neurons representing the Lateral Nucleus (LA) receives sensory information from the cortex (this term in the model will refer to both thalamus and sensory cortex), and learns to predict fear, based on these sensory inputs. These neurons project to a population in the Central Nucleus of the Amygdala (CeLOn) whose level of activation is considered the main output of the model, representing the fear response. In cases of pavlovian learning using sensory cues, this Cortex-LA-CeLOn pathway will learn to predict US arrival based on sensory information.

In the basal nucleus of the amygdala, another population of fear neurons (BAf) receives contextual inputs from the hippocampus, sensory prediction from LA, and projects to CeLOn. This Hippocampus-BAf-CeLOn pathway allows the network to make predictions based on contextual information. Finally, a population of extinction neurons in the basal nucleus of the amygdala (BAe) receives contextual information in situation of extinction from the Infralimbic cortex (IL), and learns to predict extinction conditioning, ie when predicted US do not arrive. BAe and BAf neurons are in mutual inhibition, so that in an extinction situation, neurons in BAe can inhibit BAf fear prediction, thus inhibiting the prediction of the second pathway.

BAe neurons project to a population in the central nucleus of the amygdala, CelOff, which in turn can inhibit CelON prediction in an extinction situation, inhibiting the sensory prediction of the first pathway. Consequently, the IL-BAfCelOff pathway can inhibit both sensory and context-based predictions in a case of extinction.

Another mechanism taken into account in this model is the effect of acetylcholine (ach) concentration on the different pathways. BAf and BAe activities are enhanced by ach concentration. A higher level of acetylcholine will increase BAf and BAe activities, which will favor the second or third pathway over the first one in respectively contextual learning or extinction learning. At the opposite, a lower ach concentration can allow sensory-based predictions in LA to win over BA contextual learning or extinction.

This model is implemented in Python, and is using the DANA library for neuronal computation [?]. Since we are focusing on modeling the role of the amygdalar network in pavlovian conditioning, we did not implement neuronal dynamics for the other parts of the brain providing inputs to the network, ie cortex and thalamus for LA, hippocampus for BAf and infralimbic cortex for BAe. Each of these external populations is a vector of neurons whose activities are set according to the studied paradigm.

Each amygdalar neuron is described using the mean field formalism, which consists of two variables: the membrane potential $V$ and the firing rate $U$. As we aim at modeling pavlovian conditioning by using connectivity to induce pavlovian behaviors rather than behavior-specific equations, all of our neurons are using the same equation for $V$ and for $U$ :

$$
\frac{d V_{i}^{\text {Amyg }}}{d t}=\left(-V_{i}^{\text {Amyg }}+F\left(\Sigma_{j} W_{i j}^{\text {Amyg-Input }} * U_{j}^{\text {Input }}\right)\right) / \tau
$$

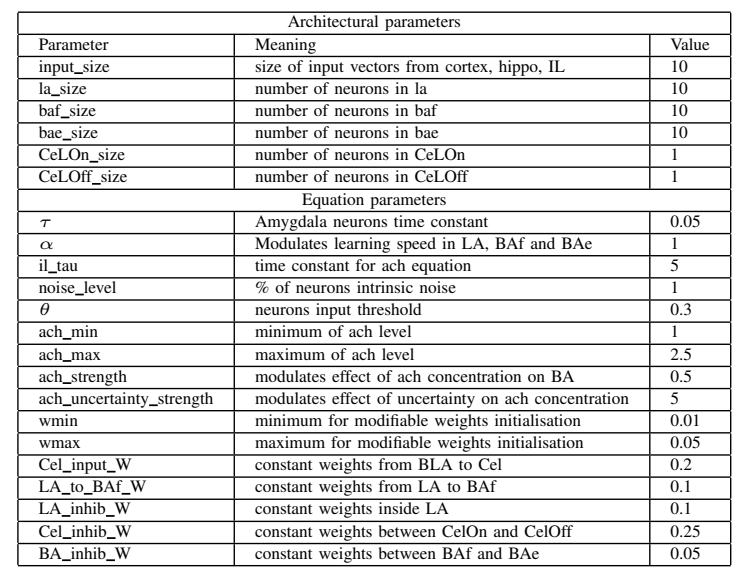

Fig. 2. Parameters describing network architecture and parameters used in activation and learning rules.

$U_{i}^{A m y g}=\operatorname{noise}\left(\operatorname{sigmoid}\left(V_{i}^{A m y g}\right)\right)-\Sigma_{k} W_{i k}^{A m y g-I n h i b} * U_{k}^{I n h i b}$

Parameters are identical for each neuron. $\tau$ is a time constant defining the dynamics of activity evolution and set to 0.05 throughout all the experiments. $\mathrm{F}$ is a non linear threshold function:

$$
F(U)=\max \left(m i n \_v a l u e, U-\theta\right)
$$

with min_value a tiny constant set to $10^{-3}$, and $\theta$ the value of the threshold, set to 0.3 .

noise () is a function which represents intrinsic neuronal noise, by using a uniform distribution centred on the value $\operatorname{sigmoid}(V)$. Results reported here are robustly obtained with an interval length of $1 \%$ of sigmoid $(V)$. Yet raising noise up to $20 \%$ does not alter network performance on the different paradigms.

Fear and extinction learning rules are implemented using hebbian learning, with a prediction error $E R R=(U S-$ $\left.U_{C e L O n}\right)$ as in the Rescorla-Wagner rule.

$$
\frac{d W_{i j}^{\text {Post-Pre }}}{d t}=E R R * U S * \alpha * U_{j}^{\text {Pre }} * U_{i}^{\text {Post }}
$$

Since extinction neurons need to increase their responsiveness when the level of prediction of the US is too high, learning rule is the opposite for extinction neurons and writes:

$$
\frac{d W_{i j}^{B A e-I L}}{d t}=-E R R * \alpha * U_{i}^{B A e} * U_{j}^{I L}
$$

where $U_{i}^{B A e}$ is the BAe postsynaptic activity, and $U_{j}^{I L}$ the presynaptic activity from IL. $\alpha$ is a learning coefficient set to 1.0 for all fear and extinction neurons.

CeLOn and CelOff neurons do not require plasticity, since their role in our model is only to integrate LA, BAf and BAe predictions, which does not need learning, at least for the paradigms tested here.

Both $U_{B A f}$ and $U_{B A e}$ are multiplicatively enhanced by ach concentration, computed as follows :

$$
\begin{aligned}
& A C h=\text { ach_strength } *(\text { baseline }+ \\
& \text { ach_uncertainty_strength } \left.* \text { noise }\left(\operatorname{sigmoid}\left(V^{A C h}\right)\right)\right)
\end{aligned}
$$




$$
d V^{A C h} / d t=\left(-V^{A C h}+F(|E R R|)\right) / \tau_{A C h}
$$

where $V^{A C h}$ is used to compute recent uncertainty which is reflected in ach concentration [14]. baseline is the baseline level of tonic acetylcholine, set to $1.0, \tau_{A C h}$ is the time constant, set to 5, $F$ is the threshold function and $E R R$ the prediction error defined above. ach_uncertainty_strength modulates the effect of the recent uncertainty $V^{A C h}$ on each concentration, and is set to 5. ach_strength modulates the overall effect of ach concentration on BA activities, and is set to 0.5 .

\section{Evaluation OF THE MODEL}

All our experiments were organized according to the protocol described in figure 3.

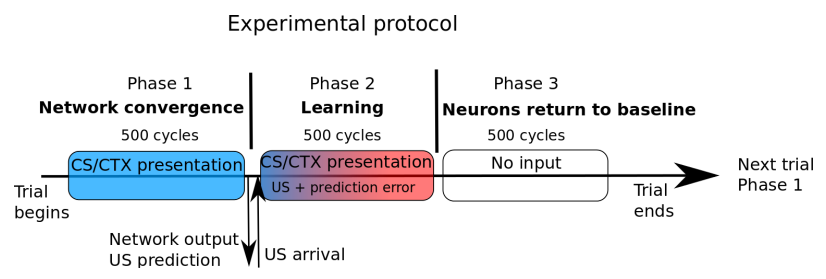

Fig. 3. Each trial is composed of three phases of identical duration. In the first phase, only the sensory CS and/or the context is shown to the network. The second phase is the learning phase, and begins with US arrival. Prediction error is computed based of the network output at the end of phase 1. During phase 3, no input is presented and amygdalar neurons return to their baseline level of activation.

One trial is a single CS-US presentation, and consists in three phases. In the first phase, the cue (CS and/or context) is presented alone to the network and enough time is given for its activities to stabilize. In the seconde phase, $\mathrm{CS}$ and/or context is maintained, and the US is presented. In the third phase, no inputs are presented and neuronal activities go down to their baseline level.
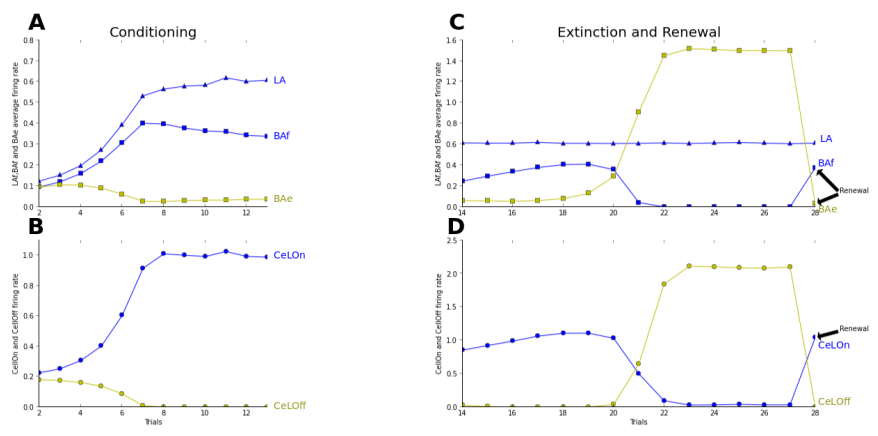

Fig. 4. Trial 1 (not shown here) was a trial without any stimulus presentation for network stabilization to baseline values. Fear conditioning takes place from trial 2 to trial 13. Fear extinction from trial 14 to trial 21. Renewal is tested in trial 22. Graphs A,B,C,D are showing average activities at the moment of US arrival. (A) Both LA and BAf activities increase during conditioning. BAe activity decreases due to BAf inhibition. (B) Due to LA and BAf increase, CeLOn activity increases, and correctly predicts US arrival after conditioning. CeLOff activity is inhibited by CeLOn (C) After a few trials, BAe activity starts to overcome BAf inhibition, and BAe strongly increases, which provokes BAf inhibition. LA firing rates remain steady. In the renewal context, IL does not provide any longer extinction information to BAe, which stops BAe firing and releases its inhibition on BAf. (D) During extinction, CeLOff activity increases with BAe increase and overcome CeLOn inhibition, still powered by LA firing. In the renewal trial, CeLOff is no longer sustained by BAe, and CeLOn activity is restored to conditioning value.
Using this procedure, our network manages to reproduce classical pavlovian acquisition, contextual extinction and renewal results as observed in [15] (cf Fig.5). We also report in figure 5 results indicating that our network nicely reproduces the blocking paradigm.
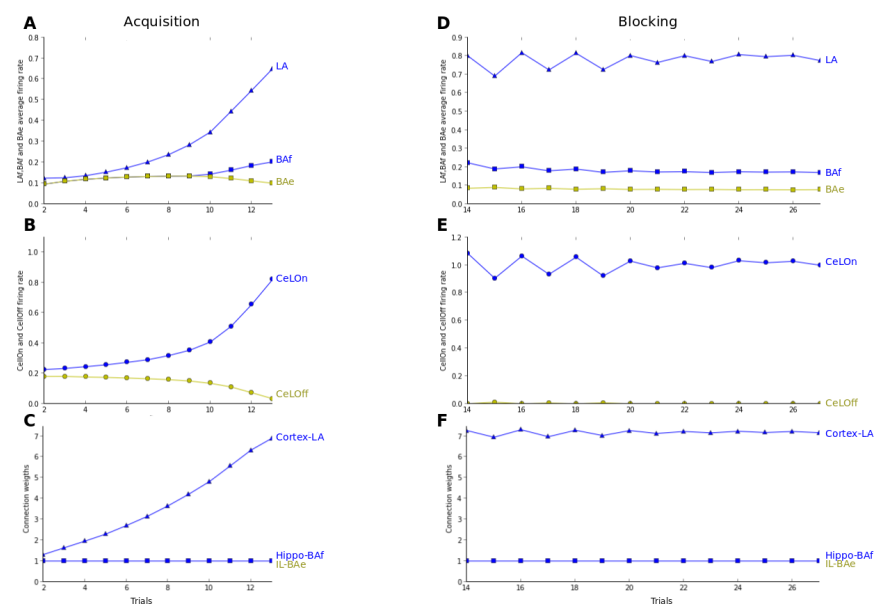

Fig. 5. Acquisition takes place from trial 2 to 13. During acquisition, a single sensory CS is presented to the network followed by US arrival (without context). Blocking takes place starting from trial 14. A new CS is presented simultaneously to the previous one, followed by US arrival. (A,B) Acquisition similar to figure 4. (C) Weights evolution during acquisition. LA weights are increasing during acquisition while the others remain steady. (D,E,F) Blocking occurs: since the first stimulus is already a good US predictor, no learning occurs based on the new stimulus presentation.

In all these experiments, each neuronal population shows different behavior, depending on its connections and inputs. During acquisition, both LA and BAf learn to predict US arrival, so that learning is distributed between these two populations. During extinction, the IL-BAe-CelOff pathway extinguishes the other two pathways thanks to BAe learning. Whereas BAf is inhibited by BAe, LA is still firing and predicting US arrival, but this prediction is inhibited in CeLOn by CelOff inhibition. In the last trial, we restore the acquisition context back and we can observe that renewal is immediate. Moreover, this network is performing fear conditioning, extinction and renewal with temporal characteristics similar to those observed in animals by [15]. Neuronal dynamics is also consistent with other experimental findings (learning distributed between BAf and LA, still fear prediction in LA when in an extinction process).

Advantages with our distributed US-prediction network include the facts that it is both able to make prediction of the US if one pathway is disturbed and that it can use the more adequate pathway to predict US in normal conditions. The following experiments were aimed at studying theses two advantages. In each experiment, after fear acquisition, we test independantly sensory-based prediction and context-based prediction of the US, in order to see which neural pathway has undergone the more learning (Fig.6).

In Fig.6.A, both CS and context are presented to the network for fear prediction (pairing experiment). Yet after learning, the network is predicting mostly based on sensory characteristics of the CS. This phenomenon emerges from the connections from LA to BAf. While only BAf can perform context-based learning, LA (by receiving sensory information 


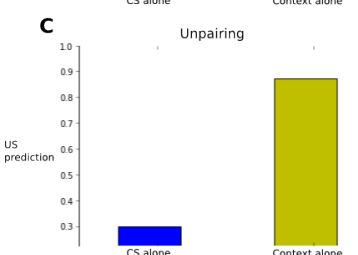

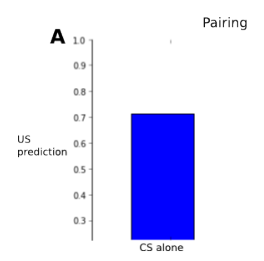

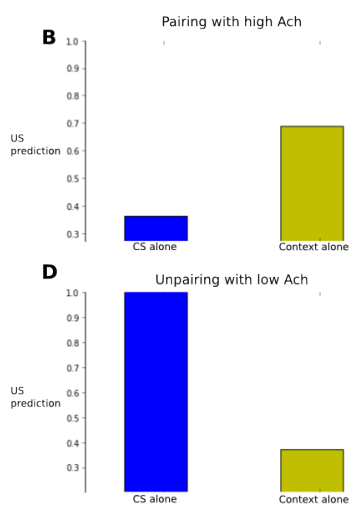

Fig. 6. CeLOn activity after learning, when only the CS (blue bar) or only the context (green bar) is presented. Similar results are observed in animals in [32].(A) During pairing experiment, both CS and context are presented as cues for US prediction. After conditioning, prediction is higher for the CS, while context-based prediction does not differ much from baseline level. (B) Pairing with increased ach level. CeLOn activity is higher for context-based prediction. ach increase causes US to be associated with context instead of CS. (C) During the unpairing experiment, both CS and context are presented to the network as cues for US prediction. Yet CS intensity is randomly varying from one trial to another, so that context, whose activity remains the same from one trial to another, is a better predictor of the US. Consequently, after learning, the sensory CS alone does not elicit a strong US prediction, while context does. (D) Unpairing experiment with ach depletion, which impairs contextual pathway learning. As a result, the sensory pathway is learning US arrival.

from cortex) and BAf (by receiving LA activations) can perform sensory-based learning. Yet, if we set ach constant to 3.0, ie. around two times the observed ach level in this paradigm, we observe the opposite phenomenon. Higher ach level means higher activities in BA, which induces a faster hebbian learning in BAf. The LA pathway does not have enough time to stabilize, so that the network is making predictions mostly based on the context (Fig.6.B), as also reported in [32].

In figure 6.C, we are also presenting both $\mathrm{CS}$ and context to the network before US arrival during the learning phase. Yet CS intensity is randomly varied from one trial to another, by multiplying it with a number randomly drawn in a uniform distribution between 0 (no CS) and 1 (normal intensity) (unpairing experiment). Thus, the context becomes a better predictor of the US than CS, since its intensity is always the same before US arrival. Figure 6.C shows that in this experiment, our network is indeed making context-based predictions. Learning takes longer, around 16 trials, which allows the measure of uncertainty to rise higher, which in turn raises ach value and allows the Hippo-BAf-CeLOn pathway to predominate in learning. Yet if we impair the BAf pathway in this experiment, by setting ach concentration to 0.5 , half of its baseline level (Fig.6.D), the network is still able to predict US arrival, by learning it with its LA pathway. If for some reason the contextual pathway is impaired, the neural network will learn to predict US arrival based on the sensory pathway, even in a paradigm in which it would normally favour the contextual one. All of these results have also been observed experimentally in animals as reported in [32].

In summary, the architecture of the neuronal network model of the amygdala that we propose can be viewed as three parallel processes and the elaboration of a criterium for arbitration. The sensory pathway including LA is the first process and tries to learn to predict the US based on sensory inputs under normal circumstances. We have explained above that by default, there is a bias favoring LA, simply due to the network connectivity. If this pathway is not performing well, either because the elemental sensory cues are not sufficient to predict US or because LA pathway is somewhat impaired, errors will accumulate, and ach level will raise, which will favor the IL-BAe-CeLOff pathway for extinction, or the HippoBAf-CeLOn pathway for contextual conditioning. The choice between the latter two processes is performed on a simple competitive process, depending on their levels of prediction. Thus ach concentration plays a critic role by monitoring recent uncertainty and enables the other two processes to predominate over the otherwise naturally chosen sensory pathway.

Moreover, as seen in the last experiment, this network structure remains flexible: if the second process (BAf pathway) is impaired, the first process through LA is still able to learn and allows the network to make sensory-based predictions. These characteristics of adaptivity and flexibility emerge from a multiple layer system, also found in animals and could not have been obtained by using a single-layer system, even with complex rules.

\section{CONCLUSION}

The main findings of this paper can be first discussed on a behavioral and evolutionary perspective. The initial goal of pavlovian mechanisms in phylogeny is to increase probability of survival by triggering rapid and automatic reflexes when the animal faces biologically significant stimuli. Along evolution, these mechanisms have evolved to incorporate more complex cases. This can be the complexification of stimuli in space and time, including attentional mechanisms in mammals. This can also be the evolution under voluntary control where CS can be seen as conditioned reinforcers or goals toward which the behavior has to be organized.

Concerning brain structure, this can also be summarized by the recent expansion of the basal nuclei [18], incorporating new inputs from the hippocampus and the prefrontal cortex and sending information about conditioned reinforcers towards the main structures of instrumental conditionning, the basal ganglia and the prefrontal cortex. But of course these new capabilities do not replace older ones (survival reflexes are still important in mammals) but just increase the size of the behavioral repertoire.

These principles are very consistent with what we observe from the characteristics of our model. In the more simple cases, the network is tuned in such a way that responses originating from LA will have a stronger effect and will trigger a fear response from basic sensory cues. Then, only in the case of error of prediction of the US, this will trigger acetylcholine, increasing the influence of BA, and the network is going to try to build more complex association from more complex sensory patterns or a longer history in time.

More generally, this bio-inspired design of a neuronal architecture is a plea for getting inspired by the modular architecture of the brain: Most of our complex cognitive functions result from the competition and collaboration of simple functional pathways. Such an architecture is more simple to build during the evolution of species but also of individuals. 
It is more resilient to damages and will integrate more easily changes by modifying local pathways instead of a unique cost function. It is certainly of highest interest for designers of Artificial Neural Networks to integrate this principle in their daily practice.

\section{REFERENCES}

[1] N. A. Schmajuk and J. J. DiCarlo, "Stimulus configuration, classical conditioning, and hippocampal function," Psychol Rev., vol. 99, no. 2, pp. 268-305, 1992.

[2] A. A. Moustafa, C. E. Myers, and M. A. Gluck, "A neurocomputational model of classical conditioning phenomena: A putative role for the hippocampal region in associative learning," Brain Res., vol. 1276, pp. 180-95, 2009.

[3] J. L. Armony, D. Servan-Schreiber, J. D. Cohen, and J. E. LeDoux, "Computational modeling of emotion: explorations through the anatomy and physiology of fear conditioning," Trends Cogn Sci, vol. 1, no. 1, pp. 28-34, Apr. 1997.

[4] N. J. Mackintosh, "A theory of attention: Variations in the associability of stimuli with reinforcement," Psychological Review, vol. 82, no. 4 pp. 276-298, 1975

[5] J. K. Kruschke, "Toward a unified model of attention in associative learning," Journal of Mathematical Psychology, vol. 45, pp. 812-863, 2001.

[6] R. S. Sutton and A. G. Barto, "Toward a modern theory of adaptive networks: expectation and prediction." Psychological review, vol. 88, no. 2, pp. 135-170, Mar. 1981.

[7] J. M. Pearce and G. Hall, "A model for Pavlovian learning: variations in the effectiveness of conditioned but not of unconditioned stimuli," Psychological review, vol. 87, no. 6, 1980. [Online]. Available: http://view.ncbi.nlm.nih.gov/pubmed/7443916

[8] L. G. Allan, "Human Contingency Judgments: Rule Based or Associative?" Psychological Bulletin, vol. 114, no. 3, pp. 435-448, 1993.

[9] R. Rescorla and A. Wagner, "A theory of pavlovian conditioning: Variations in the effectiveness of reinforcement and nonreinforcement," in Classical Conditioning II: Current Research and Theory. Appleton Century Crofts, 1972, pp. 64-99.

[10] M. A. Gluck and G. H. Bower, "From conditioning to category learning: an adaptive network model." Journal of experimental psychology. General, vol. 117, no. 3, pp. 227-247, Sep. 1988.

[11] M. Fanselow, "Contextual fear, gestalt memories, and the hippocampus," Behavioural Brain Research, vol. 110, no. 1-2, pp. 73-81, Jun. 2000

[12] C. V. Buhusi and N. A. Schmajuk, "Attention, configuration, and hippocampal function," Hippocampus, vol. 6, no. 6, pp. 621-642, Jan. 1996

[13] M. E. Le Pelley, "The role of associative history in models of associative learning: a selective review and a hybrid model." The Quarterly Journal of Experimental Psychology, vol. 57, no. 3, pp. 193-243, Jul. 2004. [Online]. Available: http://dx.doi.org/10.1080/02724990344000141

[14] A. J. Yu and P. Dayan, "Uncertainty, neuromodulation, and attention." Neuron, vol. 46, no. 4, pp. 681-692, 2005. [Online]. Available: http://dx.doi.org/10.1016/j.neuron.2005.04.026

[15] C. Herry, S. Ciocchi, V. Senn, L. Demmou, C. Muller, and A. Luthi, "Switching on and off fear by distinct neuronal circuits," Nature, vol. 454, no. 7204, pp. 600-606, Jul. 2008. [Online]. Available: http://dx.doi.org/10.1038/nature07166

[16] S. Maren, "Building and Burying Fear Memories in the Brain," The Neuroscientist, vol. 11, no. 1, pp. 89-99, Feb. 2005.

[17] R. F. Thompson and J. E. Steinmetz, "The role of the cerebellum in classical conditioning of discrete behavioral responses." Neuroscience, vol. 162, no. 3, pp. 732-755, Sep. 2009.

[18] R. N. Cardinal, J. A. Parkinson, J. Hall, and B. J. Everitt, "Emotion and motivation: the role of the amygdala, ventral striatum, and prefrontal cortex," Neuroscience \& Biobehavioral Reviews, vol. 26, no. 3, pp. 321-352, 2002. [Online]. Available: http://dx.doi.org/10.1016/s01497634(02)00007-6
[19] S. K. Barot, Y. Kyono, E. W. Clark, and I. L. Bernstein, "Visualizing stimulus convergence in amygdala neurons during associative learning," Proceedings of the National Academy of Sciences, vol. 105, no. 52, pp. 20 959-20963, Dec. 2008.

[20] M. D. Cassell, L. J. Freedman, and C. Shi, "The Intrinsic Organization of the Central Extended Amygdala," Annals of the New York Academy of Sciences, vol. 877, no. 1, pp. 217-241, Jun. 1999.

[21] H. Eichenbaum, M. Sauvage, N. Fortin, R. Komorowski, and P. Lipton, "Towards a functional organization of episodic memory in the medial temporal lobe," Neurosci Biobehav Rev., vol. 36, no. 7, pp. 1597-1608, 2012.

[22] M. Meeter, C. E. Myers, and M. A. Gluck, "Integrating Incremental Learning and Episodic Memory Models of the Hippocampal Region," Psychological Review, vol. 112, no. 3, pp. 560-585, Jul. 2005.

[23] R. C. O'Reilly and J. W. Rudy, "Conjunctive Representations in Learning and Memory: Principles of Cortical and Hippocampal Function," Psychological Review, vol. 108, no. 2, pp. 311-345, 2001

[24] J. M. Fuster, "The Prefrontal Cortex - An Update: Time Is of the Essence," Neuron, vol. 30, no. 2, pp. 319-333, 2001.

[25] J. D. Wallis, "Orbitofrontal cortex and its contribution to decisionmaking." Annual review of neuroscience, vol. 30, no. 1, pp. 31-56, Apr. 2007.

[26] D. Sierra-Mercado, N. Padilla-Coreano, and G. J. Quirk, "Dissociable roles of prelimbic and infralimbic cortices, ventral hippocampus, and basolateral amygdala in the expression and extinction of conditioned fear." Neuropsychopharmacology, vol. 36, no. 2, pp. 529-538, Jan. 2011.

[27] W. M. Pauli, T. E. Hazy, and R. C. O'Reilly, "Expectancy, Ambiguity, and Behavioral Flexibility: Separable and Complementary Roles of the Orbital Frontal Cortex and Amygdala in Processing Reward Expectancies," Journal of Cognitive Neuroscience, vol. 24, no. 2, pp. 351-366, 2011. [Online]. Available: http://dx.doi.org/10.1162/jocn_a_00155

[28] M. Jan, "Emotion and learning : a computational model of the amygdala," Ph.D. dissertation, Lund University Cognitive Studies, 2002.

[29] W. M. Pauli and R. C. O'Reilly, "Attentional control of associative learning-a possible role of the central cholinergic system," Brain Research, vol. 1202, pp. 43-53, Apr. 2008.

[30] J. LeDoux, "The amygdala," Current Biology, vol. 17, no. 20, pp. R868R874, Oct. 2007.

[31] S. Lee, S.-J. Kim, O.-B. Kwon, J. H. Lee, and J.-H. Kim, "Inhibitory networks of the amygdala for emotional memory," Frontiers in Neural Circuits, vol. 7, no. 129, 2013. [Online]. Available: http://www.frontiersin.org/neural_circuits/10.3389/fncir.2013.00129/abstract

[32] L. Calandreau, P. Trifilieff, N. Mons, L. Costes, M. Marien, A. Marighetto, J. Micheau, R. Jaffard, and A. Desmedt, "Extracellular hippocampal acetylcholine level controls amygdala function and promotes adaptive conditioned emotional response." The Journal of neuroscience : the official journal of the Society for Neuroscience, vol. 26, no. 52, pp. 13556-13566, Dec. 2006.

[33] S. Ciocchi, C. Herry, F. Grenier, S. B. E. Wolff, J. J. Letzkus, I. Vlachos, I. Ehrlich, R. Sprengel, K. Deisseroth, M. B. Stadler, C. Muller, and A. Luthi, "Encoding of conditioned fear in central amygdala inhibitory circuits," Nature, vol. 468, no. 7321, pp. 277-282, Nov. 2010. [Online]. Available: http://dx.doi.org/10.1038/nature09559 